Historic, Archive Document

Do not assume content reflects current scientific knowledge, policies, or practices. 



\title{
GEORGE L. EHRLE
}

BROAD STREET, Near Allwood Road,

$$
\text { RICHFIHLD, N. J. }
$$

Clifton R. D. No.

'Phone Passaic 7811

\author{
SPRING 1927 \\ IVholesale Price List \\ To the Nursery Trade
}

This price list does not cover everything I grow, but only such stock as I have to offer in quantity. I have been adding a number of the choicest plants from European Nurseries, which I will offer as soon as stock is available. Every square foot of my nursery is under irrigation.

SHIPPING FACILITIES: All plants are forwarded by Express or Parcel Post, the purchaser paying charges. I make no deliveries. For' Parcel Post Charges, add ten per cent, extra, to all orders East of the Mississippi and twenty per cent. West of the Mississippi.

REMITTANCES: Should be made by Post Office Money Order, Express Money Orders or drafts on New York Banks. Please send sufficient money with order to cover the whole bill. I decline to send plants Collect on Delivery, and disclaim all responsibility when remittances are not made as above directed. A convenient method of remitting for small accounts is in one and two cent postage stamps. Money should not be sent by mail.

PACKING AT COST: No charge is made for packing when cash accompanies the order.

TERMS: Strictly Cash.

While I may exert the greatest care to have everything true to name I winl not assume ary responsibility for errors that naly occur other than to replace any stock that nay prove untrie.

\section{Faithfully yours,}

GEORGE L. EHRLE.

\begin{tabular}{|c|c|c|}
\hline & 10 & 100 \\
\hline Achillea Boule de Neige & $\$ 1.00$ & $\$ 8.00$ \\
\hline millefolium roseum & 1.00 & 8.00 \\
\hline Aconitum Fischerii, $\$ 100.00$ a 1,000 . & 2.00 & 12.00 \\
\hline Spark's Variety, $\$ 100.00$ a 1,000 & 2.00 & 12.00 \\
\hline Alyssum luteum & 1.00 & 8.00 \\
\hline saxatile & 1.00 & 8.00 \\
\hline Anchusa Italica Dropmore & 1.00 & 8.00 \\
\hline Anemone pulsatilla & 1.00 & 8.00 \\
\hline Anthemis tinctoria Kel. & .90 & 7.00 \\
\hline Aquilegia, long spurred & -1.00 & 8.00 \\
\hline chrysantha & 1.00 & 8.00 \\
\hline coerulea & 1.25 & 10.00 \\
\hline rosea $\ldots . . .$. & 1.25 & 10.00 \\
\hline Mrs. Scott Elliott's Strain & 1.00 & 8.00 \\
\hline Armeria mar, Laucheana .......... & 1.00 & 8.00 \\
\hline Artemisia lactifiora & 1.50 & 12.00 \\
\hline Asclepias tuberosa .... & .80 & 6.00 \\
\hline Asters, Sub-coerulea & .75 & 6.00 \\
\hline Novae Angliae. & .80 & 6.00 \\
\hline rosea $\ldots . . .$. & .1 .00 & 8.00 \\
\hline Arabis alpina. & 1.00 & 8.00 \\
\hline fl. pl. & 1.85 & 10.00 \\
\hline Boltonia asteroides & 1.00 & 8.00 \\
\hline latisquama ....... & 1.00 & 8.00 \\
\hline nana & 1.00 & 8.00 \\
\hline Campanula carpatica & 1.00 & 8.00 \\
\hline glomerata & 1.25 & 10.00 \\
\hline media and calycantha & .75 & 6.00 \\
\hline Centaurea montana & 1.25 & 10.00 \\
\hline Cerastium tomentosa & 1.00 & 8.00 \\
\hline Chelone Lyoni & 1.25 & 10.00 \\
\hline Chrysanthemum, strong divisions, 12 named varieties ...... & -1.00 & 8.00 \\
\hline Cimicituga racemosa & 1.00 & 8.00 \\
\hline Coreopsis lanceolata gd. fl. ............. & 1.00 & 8.00 \\
\hline Delphinium Belladonna improved & 1.00 & 8.00 \\
\hline hybrids & -1.25 & 10.00 \\
\hline 3 yr. old clumps & -2.00 & 15.00 \\
\hline $\begin{array}{l}\text { Lamartine, One of the finest Delphinimm for cutting } \\
\text { needs no staking. Dark blue with white eye, } 3 \text { to }\end{array}$ & & \\
\hline Dianthus Carmen, double pink & $\begin{array}{l}3.50 \\
1.00\end{array}$ & $\begin{array}{r}20.00 \\
8.00\end{array}$ \\
\hline Furst Bismarck & 2.00 & 15.00 \\
\hline Her Majesty, double white & . 1.00 & 8.00 \\
\hline
\end{tabular}


Dianthus, Continued

Lord Lyon, double rose …........................................ 1.00

Mr's. Simkins, double white 1.00

Miss G. Cranfield, new single pink ............................ 1.00

Rose de Mai, double pink

White Reserve, double white .....................................

Dicentra spectabilis ................... 3.00

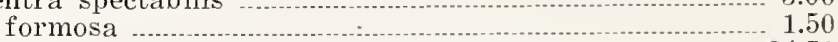

Dictamnus Caucasicus, strong 5 yr. plants ..................... $\$ 4.50$

Digitalis gloxinoides, pink, purple and white, separate

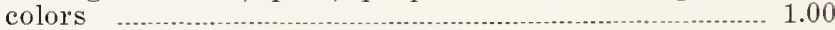

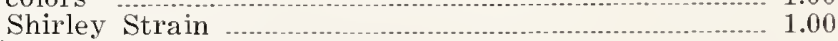

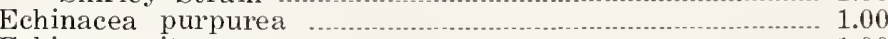

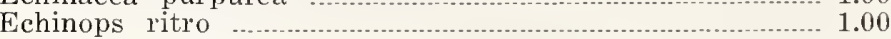

Eryngium planum ........................................................... 1.00

Eulalia Japonica 1.25

Eupatorium ageratoides

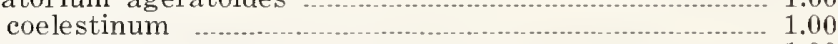

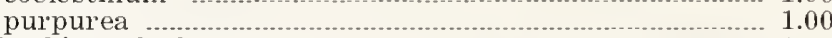

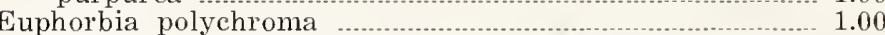

Funkia undulata variegata, $4 \mathrm{c}$ an eye; $\$ 35.00$ a 1,000 eyes

Gaillardia grandiflora

Gaillardia grandiflora $\ldots \ldots \ldots \ldots \ldots \ldots$
Geum Heldrichii

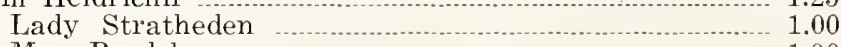

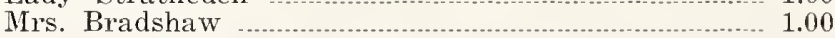

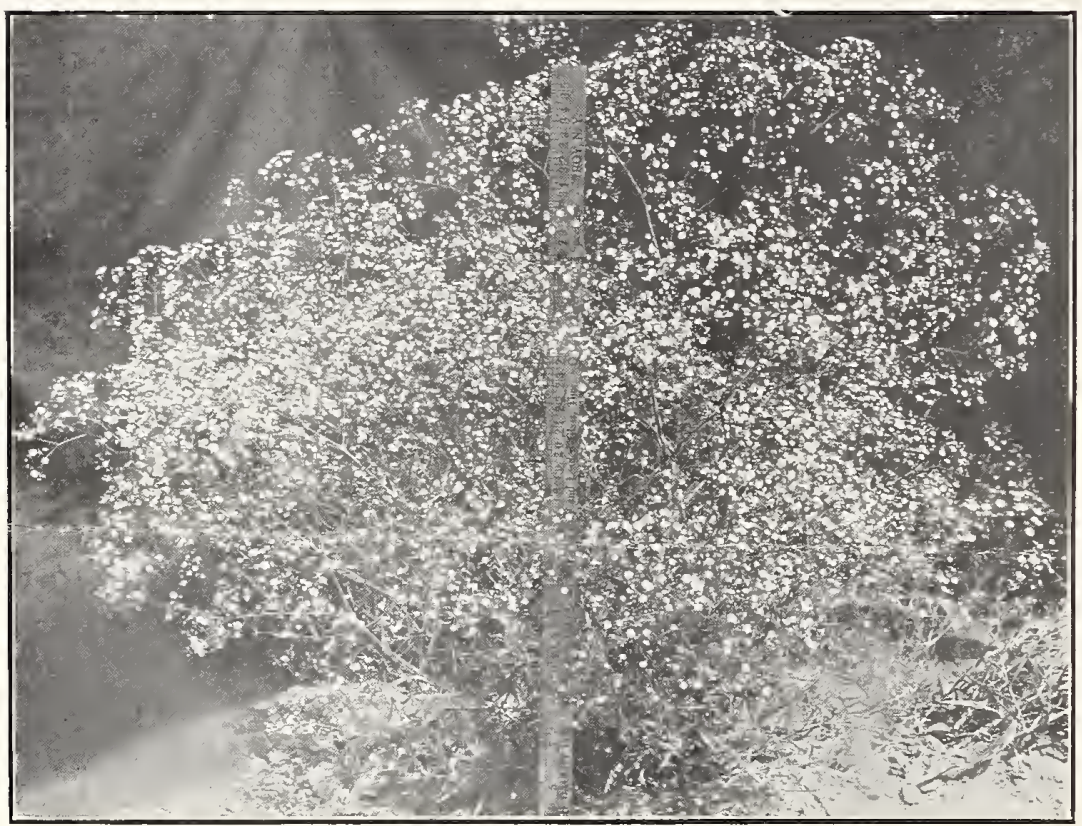

Gypsophila Ehrlei, Two Year Old Plant.

Gypsophila Ehrlei surpasses any that has ever been introduced. It is a distinct new form, early, pure white, double-flowering and not a variety of the plentiful Gypsophila paniculata fl. pl. An additional feature of this novelty is that its largest crop of flowers is produced before paniculata begins to bloom. After the main crop has been cut, flower spikes still make their appearance until frost. It is far superior to Gypsophila pan. fl. pl. because of the enormous size of its pure white flowers. Flowers were cut as late as November 15th. Plants at the present time still have green tops. Send for my pamphlet. All orders will be filled in rotation. Strong field grown, grafted plants. 5 to $25, \$ 1.00$ each; 25 to $100, \$ 75.00$ a hundred.

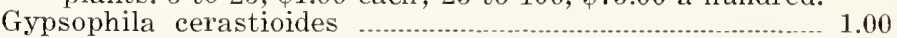

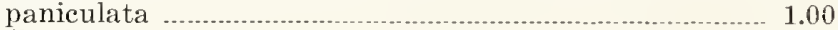

fl. pl., strong grafted, 1 yr. old, field grown ........ 3.50 repens 1.00

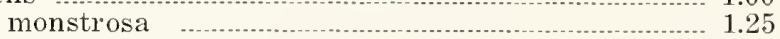

Helenium autumnale

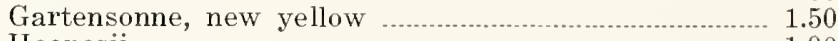
Hoopesii Riverton Gem

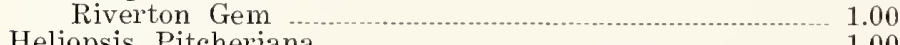

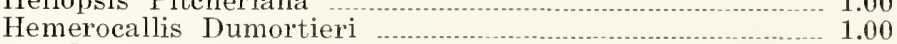
flava 1.00 luteola

Middendorfi (Dr. Regal's) 1.00 Thunbergii (Dr. Regal's) …..................................

Hepatica triloba

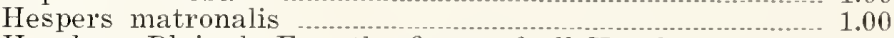

Heuchera Pluie de Feu, the finest of all Heucher'a strong $\begin{array}{ll}\text { divisions } & 2.50 \\ \text { sanguinea La Perle, dark red } & 2.50\end{array}$

Hollyhocks, Chater's double white yellow, pink, red, $\$ 50.00$ a 1,000 


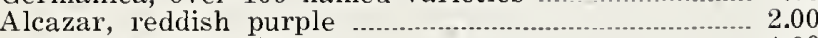

Candelabre, lavender .................................................. 1.00

Celeste, light blue ......................................................... 1.00

Cherubim, lilac ....................................................... 1.00

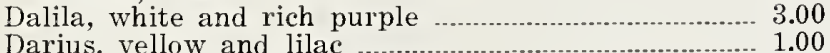

Darius, yellow and lilac $\ldots$

Eldorado, bronze and lavender ................................... 1.00

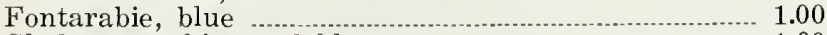

Gladstone, white and blue ............................................ 1.00

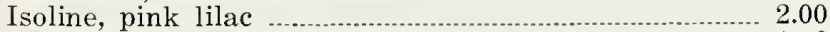

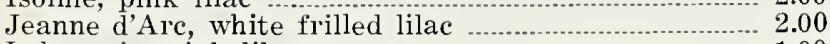

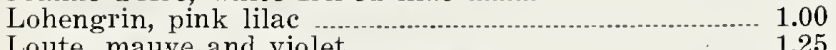

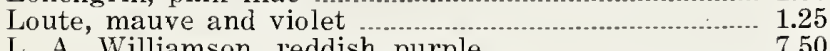

L. A. Williamson, reddish purple ............................... 7.50

Mary Garden, yellow and lavender .............................. 2.00

Mme. Chereau, white frilled blue ............................ 1.00

Mme. Guerville, white frilled blue ....................... 1.00

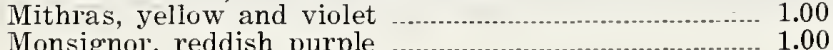

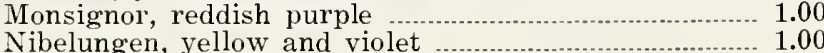

Pallida Dalmatica, $\$ 75.00$ a 1,000 ; pale blue ............... 1.00

Perfection, dark blue 1.00

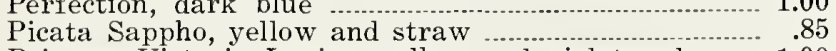

Princess Victoria Louise, yellow and violet red ........ 1.00

Quaker Lady, smoky lavender ................................ 2.00

Queen Alexandra, pink lilac ....................................... 1.00

Queen of May, pink ................................................. 1.00

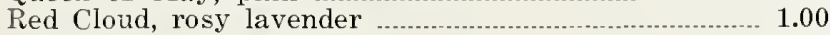

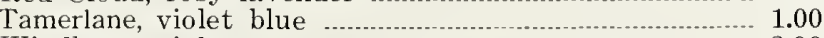

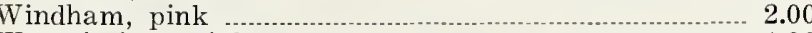

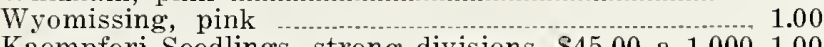

Iris Kaempferi Seedlings, strong divisions, $\$ 45.00$ a $1,0001.00$

Gold Bound, double white

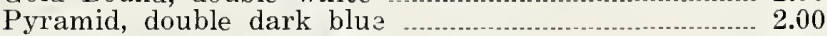

Iris Pumila, blue, yellow, $\$ 50.00$ a $1,000 \ldots$

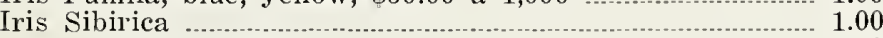

Perrys blue ........ 6.00

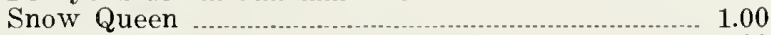

Lavendula vera $\ldots \ldots$

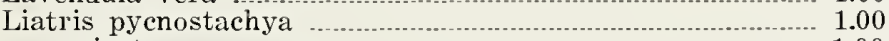

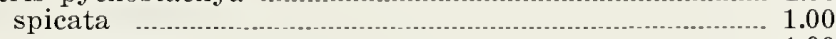

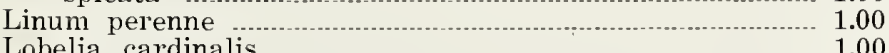

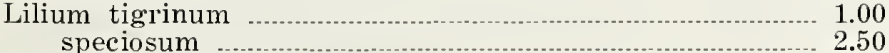

$\begin{array}{cc}\text { speciosum } & 2.50 \\ \text { inus polyphyllus : } & 1.00\end{array}$

Lupinus polyphyllus
alba

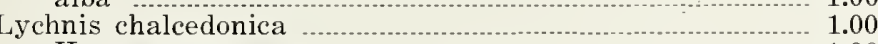

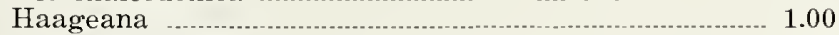

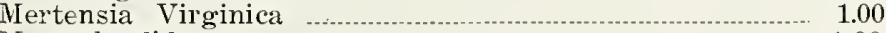

Monarda didyma, rosea

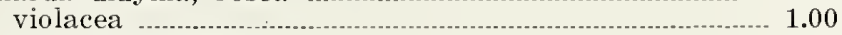

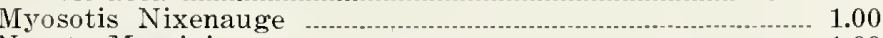

Nepeta Mussini ..... 1.00

Oenothera Fraseri .......

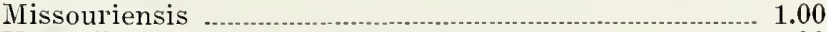

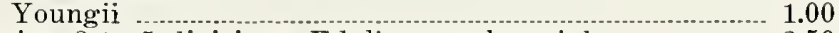

Peonies, 3 to 5 divisions, Edulis superba, pink

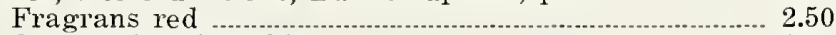

Queen Victoria, white ............................................. 2.50

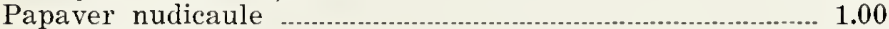

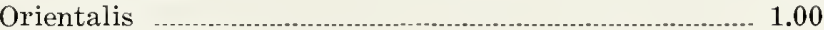

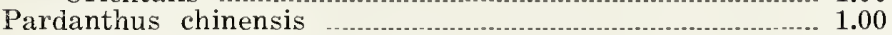

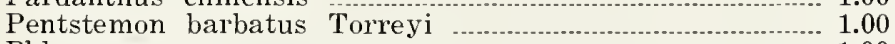

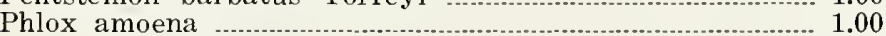
Canadensis $\quad 1.00$

alba grandiflora

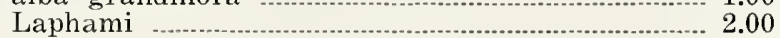

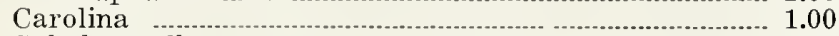

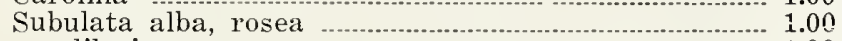

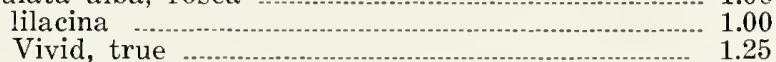

$1.25 \quad 10.00$

PHLOX, new, the best of all European introductions.

These wili be the standard varieties:

Baron van Heekeren .............................................. 2.00

Beacon

B. Comte

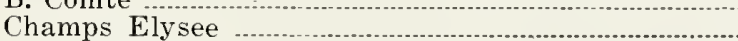

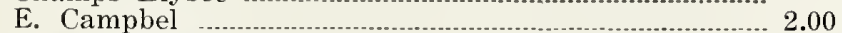

15.00

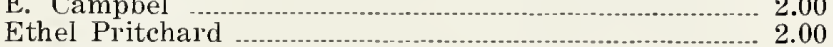

15.00

15.00

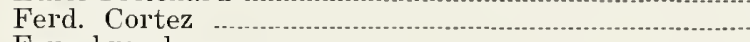

Feuerbrand

F. A. Buchner

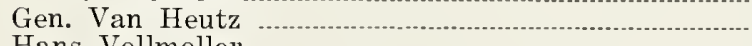

Hindenburg

15.00

15.00

Jules Sandeau 
Phlox, Continued

Rynstrom

Septemberglow

15.00

Sommerkleid

15.00

Thor

ICE except where noted

10.00

$\$ 75.00$ a 1,000 .

Physalis Franchetti, $\$ 45.00$ a $1,000 \ldots 1.00$

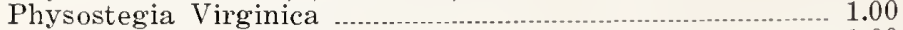
alba

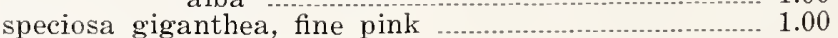

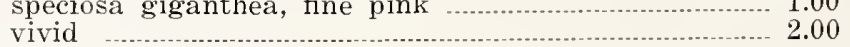

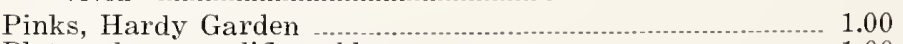

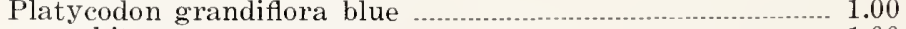

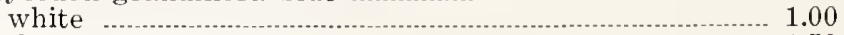

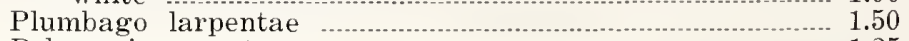

Polemonium reptans

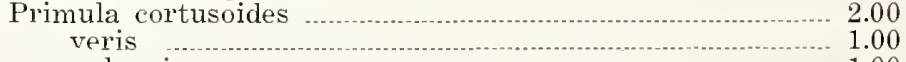

vulgaris

Pyrethrum single assorted

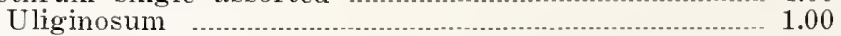

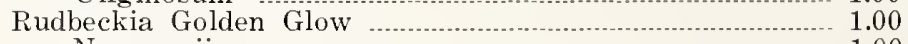

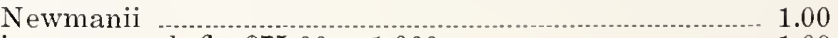

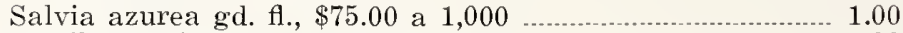

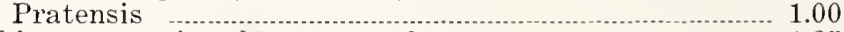

Scabiosa caucasica, $\$ 75.00$ a $1,000 \ldots \ldots \ldots$

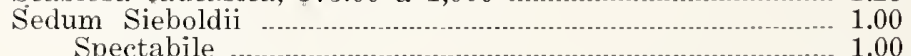

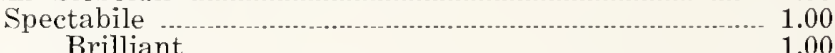

Sempervivum Tectorum …............................................... 1.00

Shasta Daisy

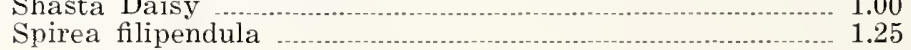
fl. pl.

ulmaria fl. pl. _.................................................. 1.50

Statice latifolia …............... 1.00

Stokesia cyanea and alba .................................................... 1.00

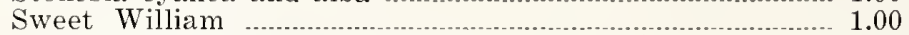

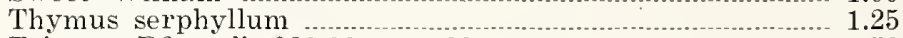

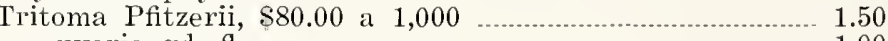

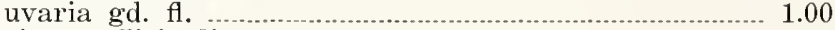

Valeriana officinalis ...... 1.00

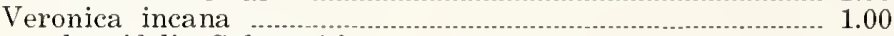

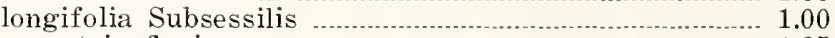

rupestris flexiosa

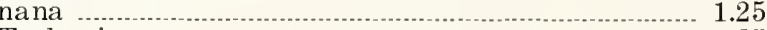

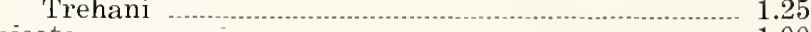

spicata
rosea $\ldots \ldots \ldots \ldots \ldots$

rosea
erica

Vinca minor

8.00

8.00

8.00

15.00

8.00

8.00

8.00

12.00
10.00

15.00

8.00

8.00

8.00
8.00

8.00

8.00

8.00

8.00

10.00

8.00
8.00

8.00

8.00

8.00

10.00

15.00

12.00

8.00

8.00

8.00
10.00

12.00

8.00

8.00

8.00

8.00

10.00

10.00

10.00

8.00

10.00

15.00
8.00

Viola cornuta Jersey Gem, the best of all V. cornutas.

Flowers all summer, perfectly hardy, strong grower.

All my stock is grown from divisions and not from

seeds

2.00

15.00

Cornuta Blue Perion

lutea splendens

Papilio

$\$ 65.00$ a 1,000

8.00

White Perfection

G. Wermig

Mauve Queen

Primrose Dame

10.00

8.00

8.00

10.00

ROOTS and HERBS:

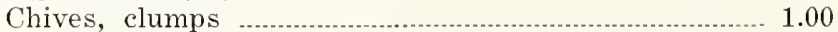

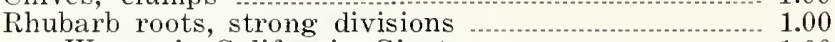

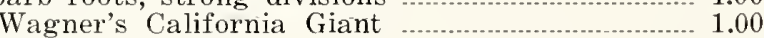

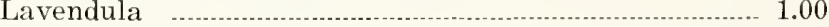

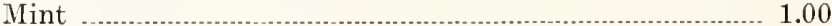

Tarragon roots, strong

BARBERRY:

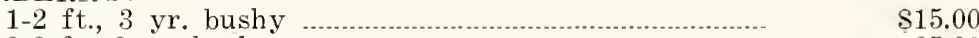

$2-3 \mathrm{ft}, 3 \mathrm{yr}$ bushy $\quad 25.00$

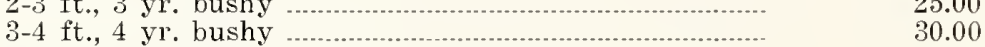

CALIFORNIA PRIVET, strong, 3 to $4 \mathrm{ft}$. ................................... 50

strong, 4 to $5 \mathrm{ft}$. 8.00

extra heavy, 10 to 15 branches, 4 to $5 \mathrm{ft}$. cannot be 10.00

Globe shaped, $\$ 1.00$ to $\$ 1.50$ each.

CLIMBING VINES:

Clematis Paniculata, strong 2 yrs. .............................. $2.00 \quad 15.00$

3 yrs. $\ldots . . \cdots-18.00$

4 yrs. .......................... $3.00 \quad 25.00$

Wisteria Sinensis multijuga, strong 4 yrs. $\quad 4.00 \quad 35.00$

Lilac, 3 to 6 in. $\$ 15.00$ a 1000

6 to 8 in. 8 . 35.00 a 1000

Korean, New, 3 to 6 in. 15.00 a 1000

Catalpa Bungei, 5-6 ft., $\$ 1.00$ to $\$ 1.50$ each.

Rosa multiflorum, 2 to 4 MM

Shrubs, assorted, best kinds $\$ 25.00$ to $\$ 50.00$ a 100

Buddleia magnifica

Thuja occidentalis, $1-2 \mathrm{ft}$ 


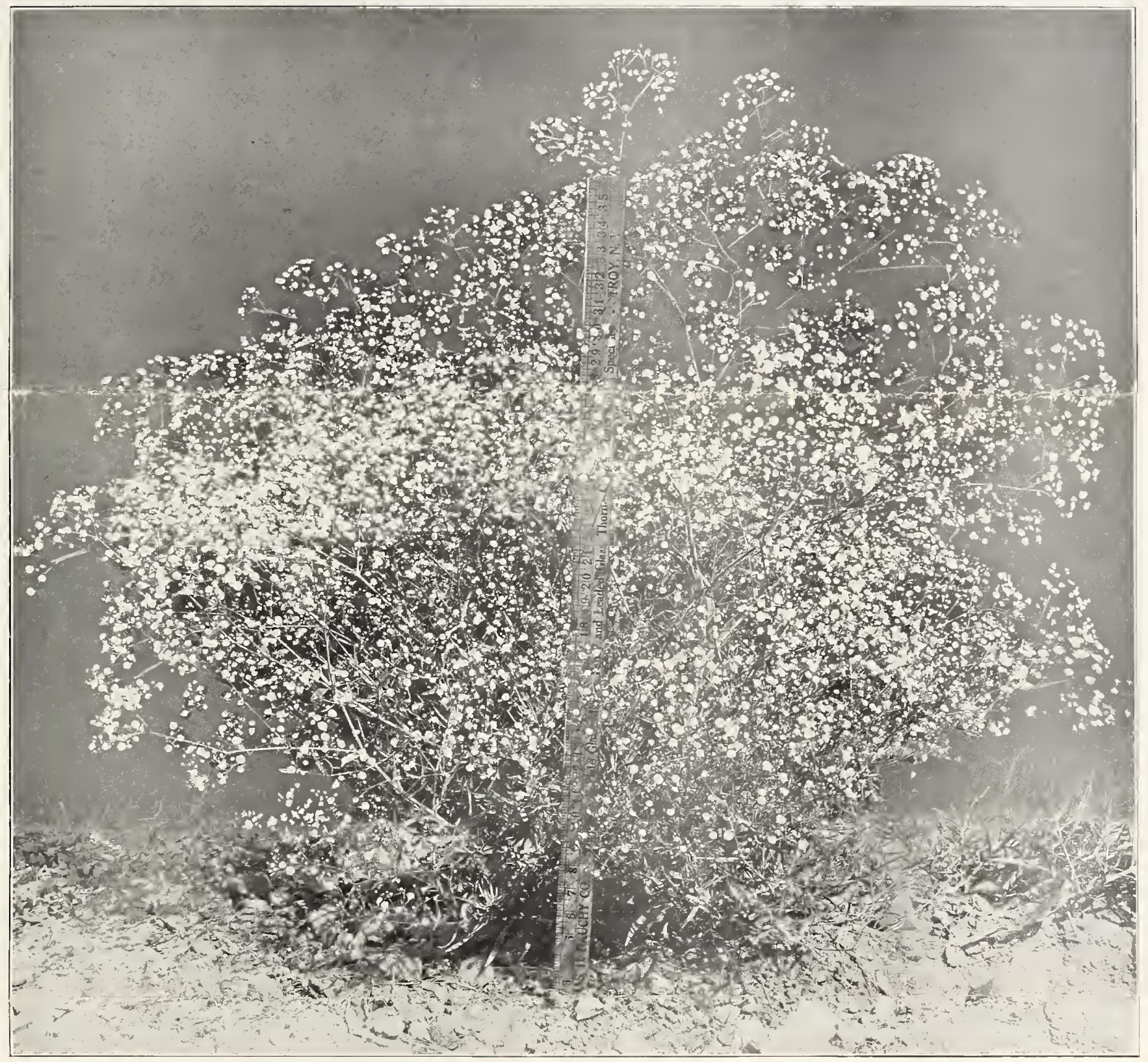

\section{GRPSOPHILA EHRLEI}

Two Year Old Plant 



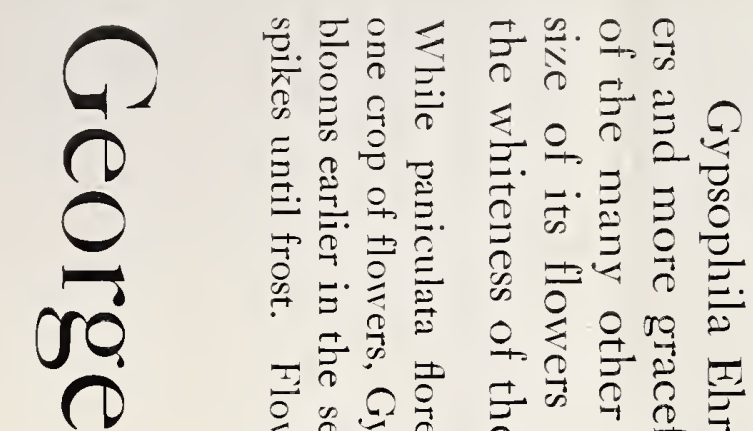

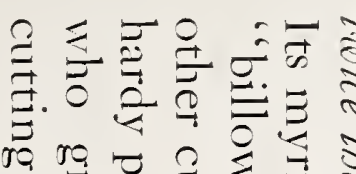

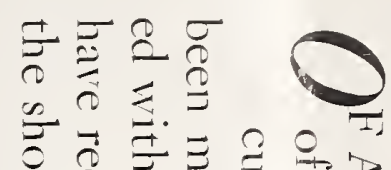

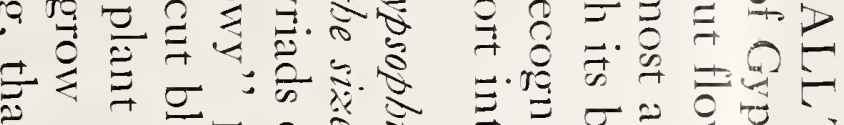

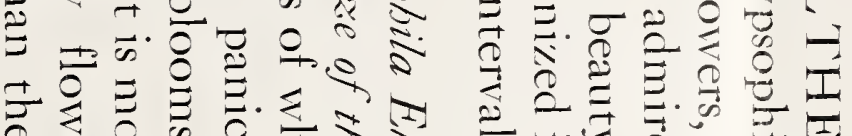

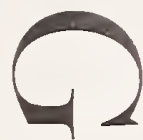

gंक

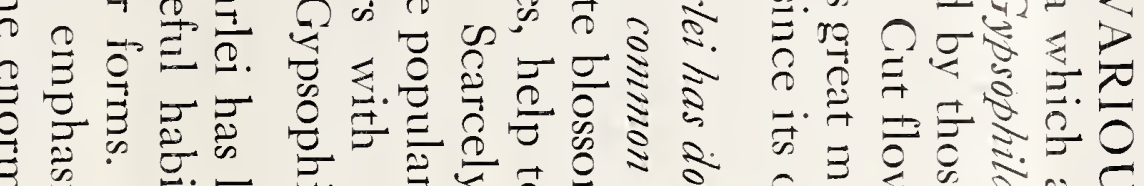

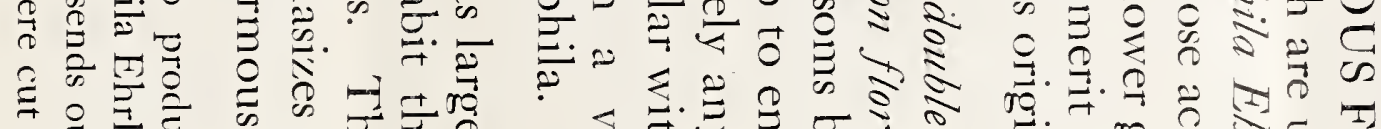

T단

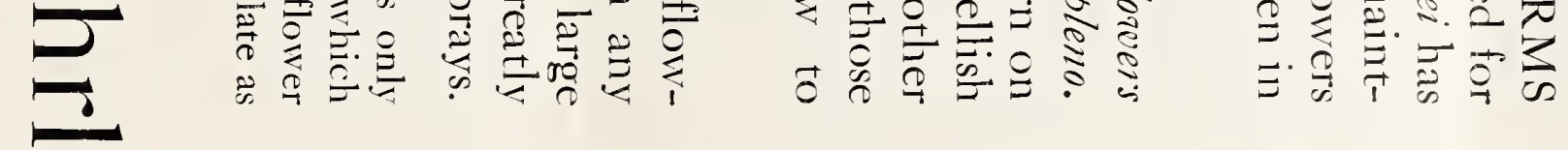

o

룽.

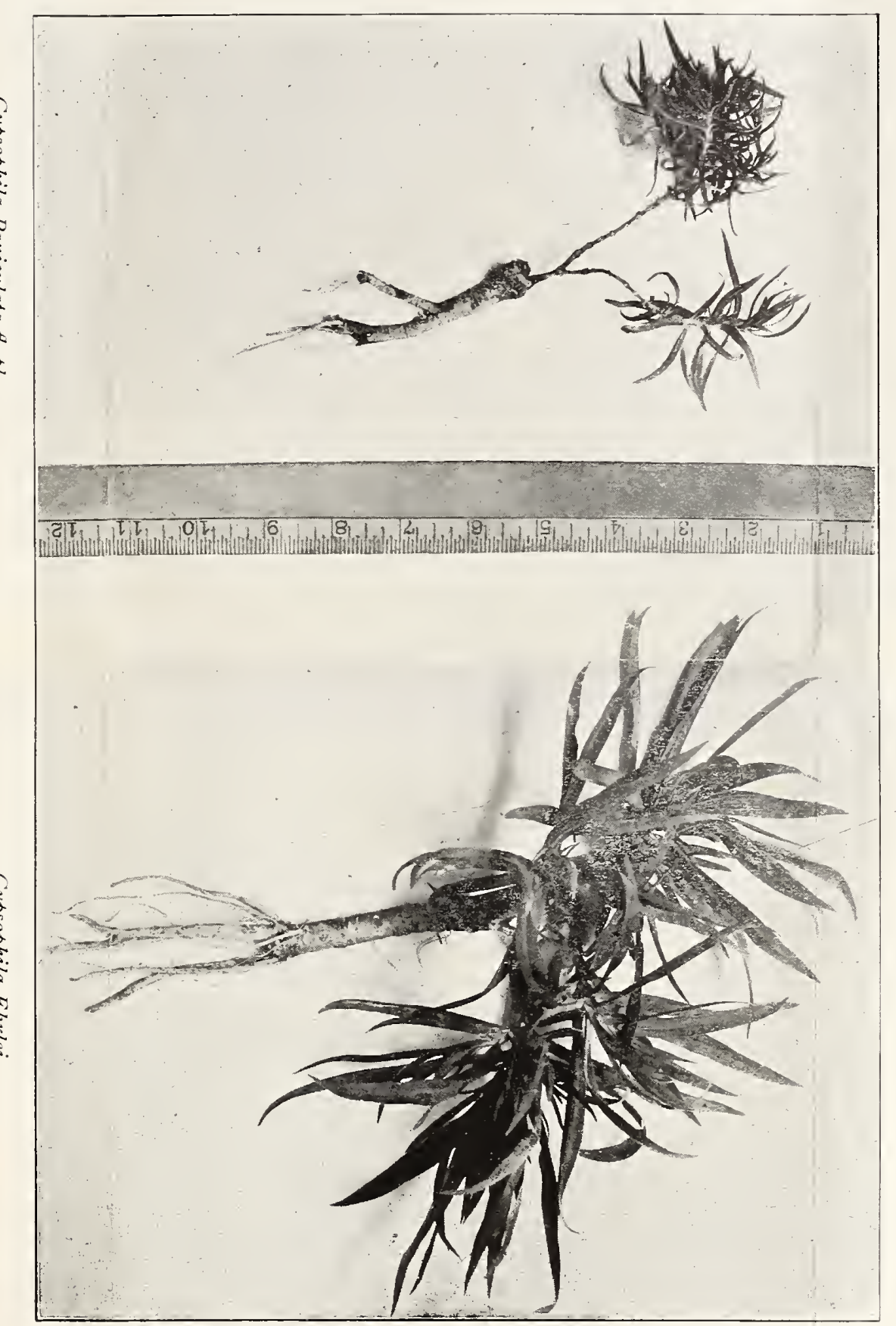

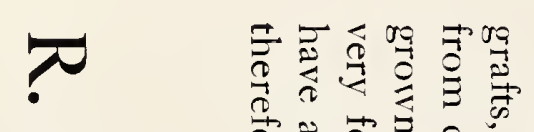

$\frac{1}{2}$

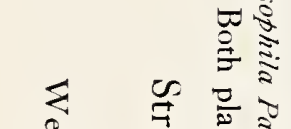

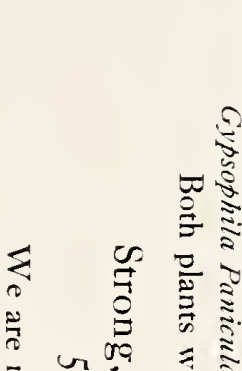

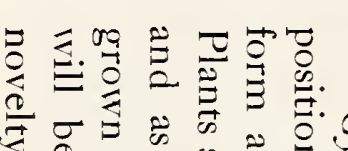

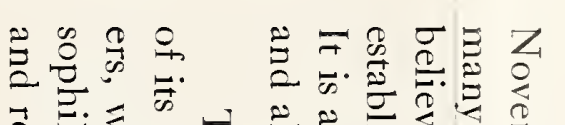

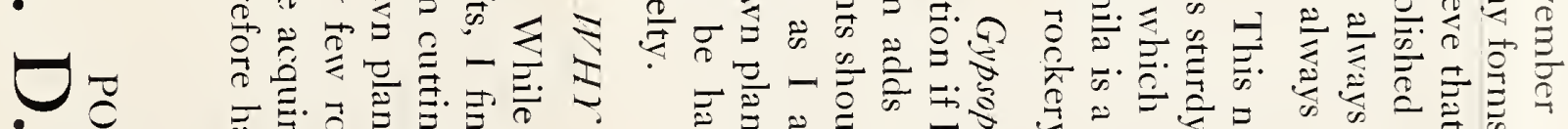

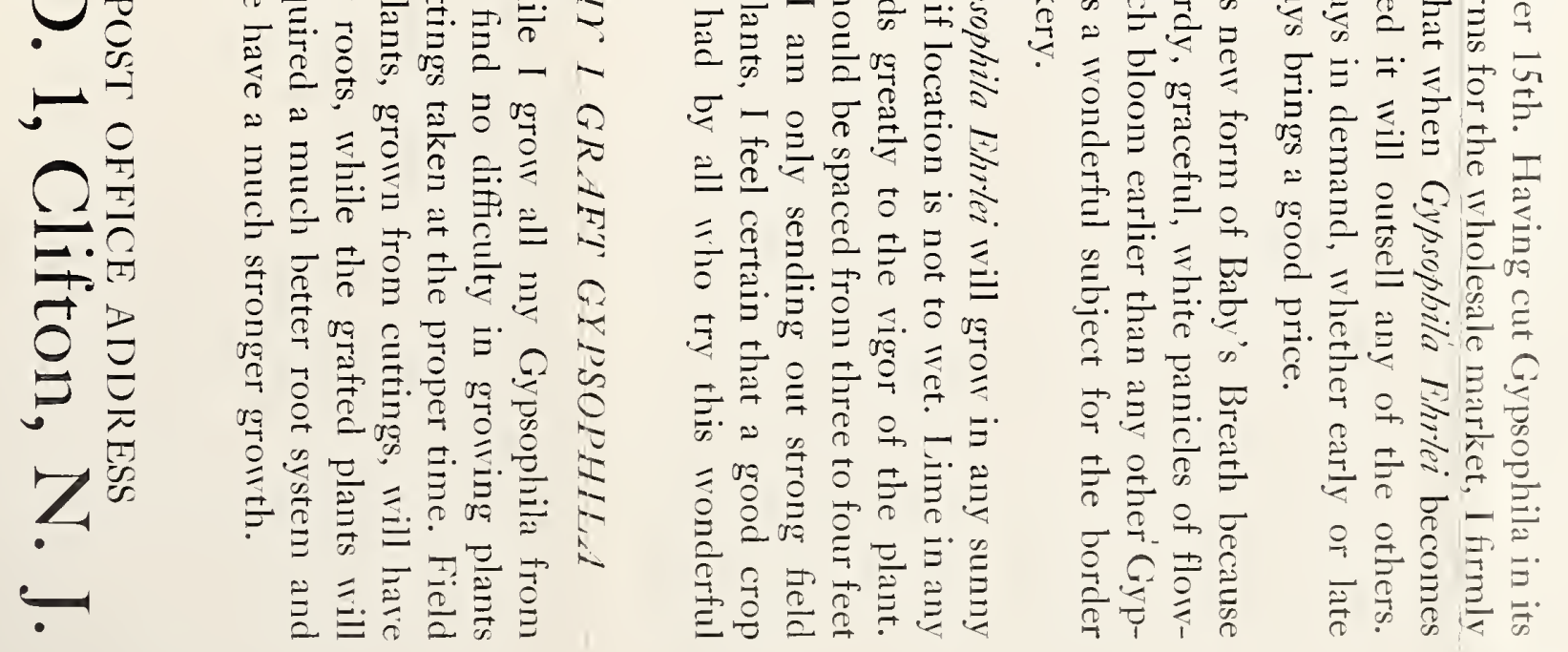
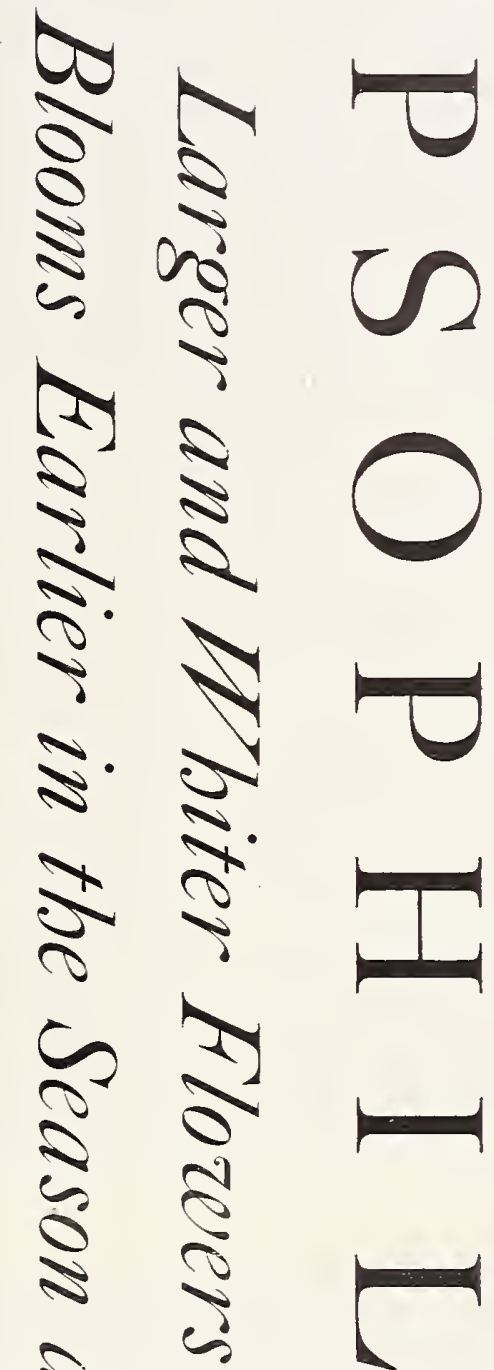

₹

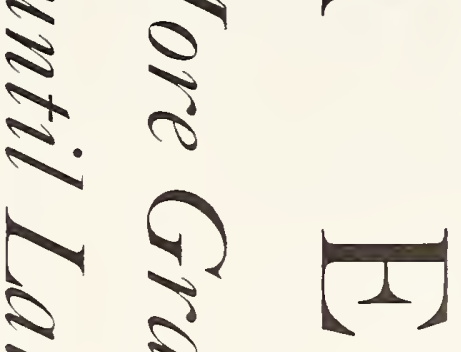

$\gamma^{2}$

i.

$\leqslant \frac{1}{2}$

$\approx N$.

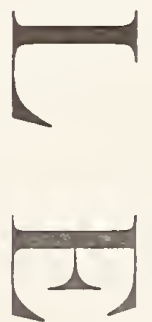




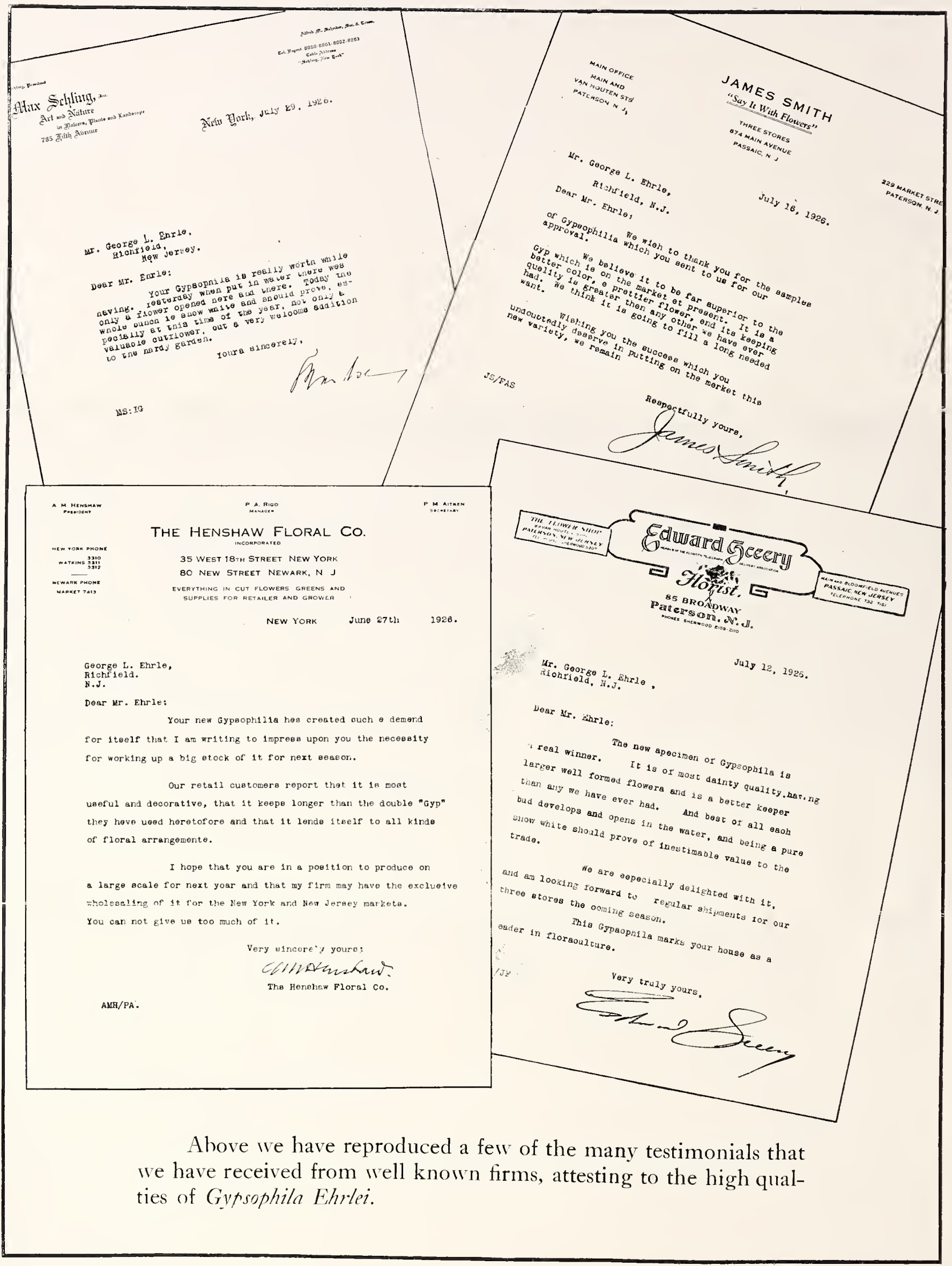

